YURI, J.E.; RESENDE, G.M.; MOTA, J.H.; SOUZA, R.J.; RODRIGUES JÚNIOR, J.C. Comportamento de cultivares e linhagens de alface americana em Santana da Vargem (MG), nas condições de inverno. Horticultura Brasileira, Brasília, v. 22, n. 2, p. 322-325, abril-junho 2004.

\title{
Comportamento de cultivares e linhagens de alface americana em Santana da Vargem (MG), nas condições de inverno
}

\author{
Jony Eishi Yuri ${ }^{1}$; Geraldo M. de Resende ${ }^{2}$; José Hortêncio Mota ${ }^{1}$; Rovilson J. de Souza ${ }^{1}$; Juarez C. \\ Rodrigues Júnior ${ }^{3}$ \\ ${ }^{1}$ UFLA-DAG, C. Postal 37, 37200-000 Lavras-MG; E-mail: jonyyuri@uol.com.br; ²Embrapa Semi-Árido, C. Postal 23, 56300-000 \\ Petrolina-PE; ${ }^{3}$ Refricon, Rodov. Regis Bittencourt s/n km 294, 06850-000 Itapecerica da Serra-SP
}

\section{RESUMO}

Cultivares e linhagens de alface americana adaptadas ao cultivo de inverno, foram avaliadas em experimento conduzido em Santana da Vargem (MG). Utilizou-se o delineamento em blocos casualizados, com três repetições. As massas frescas totais, de 42 genótipos variaram de 772 a $1268 \mathrm{~g} \mathrm{planta}^{-1}$. O melhor desempenho foi obtido pelas linhagens P1823-0014206-1 (1268 g planta $\left.^{-1}\right)$ e P1823-0014325-3 (1232 $\left.\mathrm{g} \mathrm{planta}^{-1}\right)$, que não diferiram estatisticamente entre si. Para a massa fresca comercial, a variação verificada foi de 378 a 729 g planta $^{-1}$. Entre os genótipos estatisticamente superiores, obteve-se variação de 561 a $729 \mathrm{~g} \mathrm{planta}^{-1}$. Houve variação de 35,6 a 49,2 cm na circunferência de cabeça comercial. A linhagem "P1823-00143253", com a maior circunferência de cabeça comercial, foi estatisticamente superior às demais $(49,2 \mathrm{~cm})$. O comprimento de caule variou de 1,9 a $4,9 \mathrm{~cm}$, valores considerados aceitáveis para o processamento industrial. Verificou-se uma variação abrangendo todos os graus da escala de notas para resistência à Bremia lactucae, sendo resistentes: SVR2210 (4,2); GX635 (4,2); P1843-1000148-1 (4,5); P1831-0015585-1 (4,5); P1831-0015338-1 (4,5); P1790011945-1 (4,7); P1814-0013283-1 (4,7); P1843-1000697-1 (4,7); P1843-1000171-1 (4,7); P1843-1000179-2 (5,0); P1843-10005721 (5,0); P1692-0001843-1 (5,0); P1831-0015110-1 (5,0); P18310015060-1 (5,0); P1831-0015583-2 (5,0); P1831-0015309-1 (5,0); P1831-0015058-2 (5,0); P1823-0014206-1 (5,0); P1831-0015036$1(5,0)$ e P1823-0014325-3 (5,0). A cultivar Raider, utilizada como padrão, mostrou-se altamente suscetível a essa doença.

Palavras-chave: Lactuca sativa L., produção, competição de cultivares, resistência ao míldio.
ABSTRACT

Performance of crisphead lettuce cultivars and inbred lines in Santana da Vargem, Brazil, under winter conditions

Crisphead lettuce cultivars and inbred lines adapted to winter cultivation were evaluated in a trial of 42 cultivars, in Santana da Vargem, Minas Gerais State, Brazil. The experimental design was of randomized complete blocks, with 3 replications. Total fresh weight varied from 772 to $1268 \mathrm{~g} \mathrm{plant}^{-1}$. The best performance was obtained from the inbred lines P1823-0014206-1 (1268 g plant $\left.^{-1}\right)$ and P1823-0014325-3 (1232 g plant $\left.^{-1}\right)$, which did not differ one from the other. The commercial fresh weight varied from 378 to $729 \mathrm{~g}$ plant $^{-1}$ and a variation of 561 to $729 \mathrm{~g} \mathrm{plant}^{-1}$ was obtained among statistically superior genotypes. The commercial head circumference varied from 35.6 to $49.2 \mathrm{~cm}$. The inbred line with the biggest head circumference (P1823-0014325-3 with $49.2 \mathrm{~cm}$ ) was statistically different from the other genotypes. Stem length varied from 1.9 to $4.9 \mathrm{~cm}$, these results being considered acceptable for industrial processing. A variation including all degrees of scale notes was obtained for Bremia lactucae resistance, being considered resistant: SVR2210 (4.2); GX635 (4.2); P1843-1000148-1 (4.5); P18310015585-1 (4.5); P1831-0015338-1 (4.5); P1790-011945-1 (4.7); P1814-0013283-1 (4.7); P1843-1000697-1 (4.7); P1843-10001711 (4.7); P1843-1000179-2 (5.0); P1843-1000572-1 (5.0); P16920001843-1 (5.0); P1831-0015110-1 (5.0); P1831-0015060-1 (5.0); P1831-0015583-2 (5.0); P1831-0015309-1 (5.0); P1831-00150582 (5.0); P1823-0014206-1 (5.0); P1831-0015036-1 (5.0) and P18230014325-3 (5.0). The control cv. Raider presented high susceptibility to the disease.

Keywords: Lactuca sativa L., yield, competition, downy mildew resistance.

(Recebido para publicação em 16 de abril de 2003 e aceito em 5 de janeiro de 2004)

\begin{abstract}
A alface (Lactuca sativa L.) é a hortaliça folhosa mais difundida atualmente, sendo cultivada em quase todos os países. Segundo a FAO (2002), no ano 2000 foram produzidos no mundo 17,28 milhões de toneladas de alface, em uma área de 791.144 ha. No Brasil o IBGE (2000), informa que no ano de 1996 houve uma produção de 311.887 toneladas de alface, no valor de 152.347 milhões de reais. Desse total, cerca de 173.000 toneladas foram produzidas no estado de São Paulo, ocupando uma área de 7.859 ha e gerando 6.360 empregos (Meirelles, 1998).
\end{abstract}

No inicio da década de 80 foi introduzido no Brasil um novo grupo de alface repolhuda crespa, conhecida como alface americana, que se diferencia dos demais por apresentar folhas externas de coloração verde-escura, folhas internas de coloração amarela ou branca, imbricadas, semelhantes ao repolho e crocantes (Yuri et al., 2002). A sua grande aceitação pelas redes de fast foods ocorreu principalmente pela capacidade de manter-se crocante, quando em contato com altas temperaturas, no interior dos sanduíches e também pela re- sistência ao transporte e capacidade de armazenamento por maior tempo (Decoteau et al., 1995). Apresenta elevados teores de vitaminas e sais minerais, além do baixo teor de calorias (Katayama, 1993).

A alface americana vem adquirindo importância crescente no país. Apenas para abastecer uma grande rede de lanchonetes, tem-se produzido aproximadamente 7.800 toneladas anualmente. Em 2001, 29,6\% (7.550 t) da alface comercializada na CEAGESP, foi representada pela americana (Conjuntural..., 2001). 
O processo produtivo brasileiro passa por uma fase em que a produtividade, a eficiência, a lucratividade e a sustentabilidade são aspectos que precisam ser levados em conta. Nesse contexto, a obtenção de novas cultivares de alface, adaptadas às condições climáticas regionais, torna-se preponderante. Até o momento, um dos maiores desafios está na seleção de cultivares com pequeno comprimento de caule, elevada circunferência da cabeça comercial e que sejam produtivas além de resistentes a doenças foliares.

Com o aumento da produção na região, mesmo em condições de outono e inverno, épocas de temperatura amena e adequada à produção, a dificuldade em produzir essa hortaliça vem aumentando, principalmente pela infestação das áreas de produção pelo fungo causador do míldio (Bremia lactucae). Esta é a principal doença da alface (Davis et al., 1997) que, em regiões com temperaturas amenas, pode provocar destruição total. O sintoma inicia-se com a formação de pequenas manchas angulares, de coloração verde-clara a amarelada, na face superior da folha. Com o desenvolvimento da doença, a coloração da parte infectada torna-se marrom. Em condições de alta umidade o fungo forma frutificações brancas na face inferior das folhas (Persley, 1994). A forma mais econômica de contrôle da doença consiste na utilização de cultivares resistentes (Davis et al., 1997).

Assim, o objetivo deste trabalho foi avaliar o nível de resistência de cultivares e linhagens de alface americana a doenças foliares associado ao rendimento, nas condições de inverno, no sul de Minas Gerais.

\section{MATERIAL E MÉTODOS}

O experimento foi conduzido no município de Santana da Vargem, sul de Minas Gerais, situado a $21^{\circ} 05^{\prime} 15^{\prime \prime}$ de latitude sul, $45^{\circ} 34^{\prime} 00^{\prime \prime}$ de longitude oeste e altitude de $850 \mathrm{~m}$. O clima da região é do tipo Cwa com características de $\mathrm{Cwb}$, apresentando duas estações definidas: seca (abril a setembro) e chuvosa (outubro a março), segundo a classificação climática de Köppen. O tipo de solo predominante na área é classifi- cado como LATOSSOLO VERMELHO Distroférrico. A análise química do solo revelou: $\mathrm{pH}\left(\mathrm{CaCl}_{2}\right)=5,0 ; \mathrm{P}=139 \mathrm{mg}$ $\mathrm{dm}^{-3} ; \mathrm{k}^{+}=192 \mathrm{mg} \mathrm{dm}{ }^{-3} ; \mathrm{Ca}^{2+}=7,0 \mathrm{cmol}$ $\mathrm{dm}^{-3} ; \mathrm{Mg}^{2+}=1,1 \mathrm{cmol}_{\mathrm{c}} \mathrm{dm}^{-3} ; \mathrm{V}(\%)=$ $65 \%$.

O delineamento utilizado foi em blocos casualizados, com três repetições, sendo os tratamentos constituídos por 42 cultivares e linhagens de alface do tipo americana: P1823-0014206-1; P18230014325-3; P1831-0015309-1; P18431000697-1; P1814-0013283-1; Rocco; SVR2772; P1843-1000179-2; P18310015338-1; GX635; P1831-0015585-1; P1831-0015583-2; 99C962; P18431000572-1; P1823-0014025-3; Rubette; P1692-0001843-1; P1790-0011945-1; P1831-0015058-2; P1843-1000171-1; Paloma; P1831-0015060-1; SVR2210; P1831-0015110-1; P1608-20635-1; RTP2020; Sandy; Ace; Legacy; Sonoma; P1871-2788; P1831-00150361; P1871-2817; PSR1115; RTP3030; Raider; P1843-1000148-1; P1871-2772; PSR1904; SVR1095; SVR1153 e PSR110. A cultivar Raider foi utilizada como testemunha, pois é o material mais plantado na região pelos produtores de alface do tipo americana, apresentando grande aceitação, pela rusticidade, bom rendimento no processamento, cultivo o ano todo, tolerância à queimadura de borda das folhas externas, ao pendoamento e ao "tip-burn" e cabeça uniforme.

A semeadura foi realizada em $08 / 05 /$ 02, em bandejas de isopor contendo 200 células, preenchidas com o substrato comercial "Plantmax HT". As mudas foram transplantadas para o campo em 07/06/02, correspondendo a 30 dias após a emergência.

O preparo do solo constou de aração, gradagem e levantamento dos canteiros a 0,20 m de altura. As parcelas experimentais tiveram $2,1 \mathrm{~m}$ de comprimento e 28 mudas, espaçadas a $0,30 \mathrm{~m}$ na linha e $0,35 \mathrm{~m}$ na entrelinha. Definiu-se como área útil da parcela, as duas linhas centrais, descartando-se as duas plantas das extremidades de cada linha, onde foram retiradas seis plantas para análise. Essa área foi previamente corrigida com calcário dolomítico, elevando a saturação por bases para 70\% (Ribeiro et al., 1999), e adubada com $65 \mathrm{~kg} \mathrm{ha}^{-1}$ de $\mathrm{N}, 600 \mathrm{~kg} \mathrm{ha}^{-1}$ de $\mathrm{P}_{2} \mathrm{O}_{5}$ e $256 \mathrm{~kg} \mathrm{ha}^{-1}$ de $\mathrm{K}_{2} \mathrm{O}$, utilizando como fontes o adubo formulado 04-30-16 e superfosfato simples. Após a incorporação dos adubos instalou-se em cada canteiro duas linhas de tubo gotejador, com emissores espaçados a cada $0,30 \mathrm{~m}$ e com vazão de $1,5 \mathrm{~L} \mathrm{~h}^{-1}$; posteriormente foram cobertos com mulching de coloração preta e 35 micras de espessura.

Após o transplante, irrigou-se a área por aspersão, diariamente, durante sete dias, até o pegamento uniforme das plantas. A seguir, a irrigação foi realizada diariamente, por gotejamento, em lâminas de $3 \mathrm{~mm} /$ dia. Juntamente com a irrigação, realizaram-se as adubações de cobertura (fertirrigação), aplicando-se $40 \mathrm{~kg} \mathrm{ha}^{-1} \mathrm{de}$ $\mathrm{Ne} 85 \mathrm{~kg} \mathrm{ha}^{-1} \mathrm{de}_{2} \mathrm{O}$, utilizando-se como fontes uréia e cloreto de potássio.

Para o controle fitossanitário adotouse o método padrão utilizado pelo produtor, com pulverizações preventivas semanais, sendo utilizados para controle de doenças oxicloreto de cobre, iprodione e procimidone, e para controle de pragas pirimicarb e piretróides.

As colheitas foram realizadas entre 06 e 09/08/02, época em que se observou para cada genótipo, o máximo desenvolvimento vegetativo, apresentando cabeças comerciais compactas. Momentos antes de se iniciar a colheita, fez-se a avaliação do estado fitossanitário das plantas, baseada em escala visual de notas, variando de 1 a 5 ( $1=$ plantas com as folhas externas altamente atacadas pelo fungo Bremia lactucae; 2=presença abundante de lesões nas folhas externas; $3=$ presença moderada de lesões nas folhas externas; 4=lesões escassas nas folhas externas e $5=$ plantas com as folhas externas sadias) (Mota et al., 2003). A massa fresca total (g planta $\left.{ }^{-1}\right)$ foi obtida cortando as plantas bem rente ao solo e pesando em balança modelo US. $15 / 5 \mathrm{da}$ marca Urano, com sensibilidade de 5 gramas. A massa fresca comercial (g planta $\left.{ }^{-1}\right)$, foi obtida pesando somente as "cabeças" comerciais, retirando-se as folhas externas. Posteriormente, com o auxílio de uma fita métrica, mediou-se a circunferência da cabeça comercial $(\mathrm{cm})$. Ainda, cortou-se a cabeça da alface ao meio, possibilitando a medição do comprimento do caule $(\mathrm{cm})$ com o auxílio de uma régua graduada. 
Tabela 1. Massa fresca total e comercial, comprimento do caule, circunferência da cabeça e sanidade das folhas externas de cultivares e linhagens de alface americana. Santana da Vargem (MG), UFLA, 2002.

\begin{tabular}{|c|c|c|c|c|c|c|c|c|}
\hline \multirow{3}{*}{$\frac{\text { Cultivar/linhagem }}{\text { P1823-0014206-1 }}$} & \multicolumn{3}{|c|}{ Massa fresca (g/planta) } & \multirow{2}{*}{$\begin{array}{l}\text { Comprimento do } \\
\text { caule }(\mathrm{cm})\end{array}$} & \multirow{2}{*}{\multicolumn{2}{|c|}{$\begin{array}{l}\text { Circunferência da } \\
\text { cabeça }(\mathrm{cm})\end{array}$}} & \multirow{2}{*}{\multicolumn{2}{|c|}{ Sanidade $^{1}$}} \\
\hline & \multicolumn{2}{|c|}{ Total } & \multirow{2}{*}{$\frac{\text { Comercial }}{729 \text { a }}$} & & & & & \\
\hline & 1268 & & & $4,1 \mathrm{~d}$ & 44,8 & c & $5,0 a$ & \\
\hline P1823-0014325-3 & 1232 & & 587 a & $2,8 \quad b$ & 49,2 & & $5,0 a$ & \\
\hline P1831-0015309-1 & 1123 & $b$ & $600 a$ & $2,9 \mathrm{~b}$ & 44,9 & $\mathrm{c}$ & $5,0 \mathrm{a}$ & \\
\hline P1843-1000697-1 & 1114 & $b$ & 682 a & $3,7 \quad c$ & 45,2 & c & 4,7 a & \\
\hline P1814-0013283-1 & 1109 & $\mathrm{~b}$ & 596 a & $3,1 \quad b$ & 44,3 & c & $4,7 \mathrm{a}$ & \\
\hline Rocco & 1092 & $b$ & 648 a & $3,5 \quad c$ & 41,3 & $d$ & 3,7 & $\mathrm{~b}$ \\
\hline SVR2772 & 1085 & $b$ & 726 a & 3,6 & 44,3 & $\mathrm{c}$ & 3,5 & $b$ \\
\hline P1843-1000179-2 & 1078 & $b$ & 617 a & $3,9 \quad d$ & 42,2 & $d$ & $5,0 a$ & \\
\hline P1831-0015338-1 & 1074 & $b$ & 612 a & $3,0 \mathrm{~b}$ & 43,6 & $\mathrm{c}$ & $4,5 \mathrm{a}$ & \\
\hline GX635 & 1072 & $b$ & 637 a & $2,8 \quad b$ & 44,9 & c & $4,2 \mathrm{a}$ & \\
\hline P1831-0015585-1 & 1059 & $b$ & 647 a & $3,4 \quad c$ & 44,5 & c & $4,5 \mathrm{a}$ & \\
\hline P1831-0015583-2 & 1059 & $b$ & 692 a & $3,6 \quad c$ & 44,2 & c & $5,0 a$ & \\
\hline $99 \mathrm{C} 962$ & 1028 & $b$ & $507 \quad b$ & $3,0 \quad b$ & 44,3 & c & 1,0 & $d$ \\
\hline P1843-1000572-1 & 1024 & $b$ & 644 a & 4,9 & 44,7 & c & $5,0 \mathrm{a}$ & \\
\hline P1823-0014025-3 & 1023 & $b$ & 665 a & $2,9 \quad b$ & 46,5 & $\mathrm{~b}$ & 1,5 & d \\
\hline Rubette & 1009 & $b$ & 724 a & $4,1 \quad d$ & 42,2 & d & 3,7 & $\mathrm{~b}$ \\
\hline P1692-0001843-1 & 997 & $b$ & 634 a & $3,4 \quad c$ & 44,1 & c & $5,0 \mathrm{a}$ & \\
\hline P1790-0011945-1 & 988 & $b$ & $636 a$ & $3,1 \quad b$ & 43,2 & c & 4,7 a & \\
\hline P1831-0015058-2 & 983 & $b$ & 617 a & $3,3 \quad c$ & 44,7 & c & $5,0 \mathrm{a}$ & \\
\hline P1843-1000171-1 & 982 & $b$ & 666 a & $3,6 \quad c$ & 46,2 & $\mathrm{~b}$ & 4,7 a & \\
\hline Paloma & 957 & c & 561 a & $2,5 \mathrm{a}$ & 41,6 & $d$ & 1,7 & c \\
\hline P1831-0015060-1 & 948 & c & 599 a & $3,1 \quad b$ & 42,9 & $\mathrm{c}$ & $5,0 \mathrm{a}$ & \\
\hline SVR2210 & 948 & c & 660 a & 4,6 & 45,9 & $\mathrm{~b}$ & $4,2 \mathrm{a}$ & \\
\hline P1831-0015110-1 & 931 & c & $471 \quad b$ & $2,1 \mathrm{a}$ & 42,5 & $d$ & $5,0 \mathrm{a}$ & \\
\hline P1608-20635-1 & 930 & c & 575 a & $3,1 \quad b$ & 44,0 & c & 2,0 & $\mathrm{c}$ \\
\hline RTP2020 & 921 & c & 590 a & $3,0 \quad b$ & 41,9 & $d$ & 1,5 & $d$ \\
\hline Sandy & 912 & c & 633 a & $3,4 \quad c$ & 42,2 & $d$ & 1,7 & $\mathrm{c}$ \\
\hline Ace & 912 & c & 706 a & 3,7 & 44,1 & $\mathrm{c}$ & 1,2 & $d$ \\
\hline Legacy & 904 & c & 671 a & 4,0 & 42,3 & $d$ & 1,7 & $\mathrm{c}$ \\
\hline Sonoma & 904 & c & $543 \quad b$ & $3,3 \quad c$ & 41,4 & $d$ & 3,2 & $\mathrm{~b}$ \\
\hline P1871-2788 & 894 & c & 565 a & $3,0 \quad b$ & 41,8 & $d$ & 1,2 & $d$ \\
\hline P1831-0015036-1 & 879 & c & $491 \quad b$ & $2,8 \quad b$ & 40,0 & d & $5,0 \mathrm{a}$ & \\
\hline P1871-2817 & 874 & c & $469 \quad b$ & $1,9 \mathrm{a}$ & 37,1 & $f$ & 1,5 & $d$ \\
\hline PSR1115 & 860 & c & $480 \quad b$ & $2,0 \mathrm{a}$ & 37,8 & e & 3,2 & $\mathrm{~b}$ \\
\hline RTP3030 & 840 & c & $378 \quad b$ & $2,4 \mathrm{a}$ & 41,4 & d & 1,5 & $d$ \\
\hline Raider & 829 & c & $534 \mathrm{~b}$ & $2,8 \quad b$ & 42,4 & $d$ & 1,7 & $\mathrm{c}$ \\
\hline P1843-1000148-1 & 826 & c & 575 a & $3,8 \quad c$ & 41,1 & $d$ & $4,5 a$ & \\
\hline P1871-2772 & 823 & c & $429 \quad b$ & $1,9 \mathrm{a}$ & 35,6 & $f$ & 2,2 & $\mathrm{c}$ \\
\hline PSR1904 & 820 & c & 577 a & $2,7 \quad b$ & 39,4 & e & 1,5 & $d$ \\
\hline SVR1095 & 817 & c & $482 \quad b$ & $2,1 \mathrm{a}$ & 38,7 & e & 1,0 & $d$ \\
\hline SVR1153 & 789 & c & $512 b$ & $2,8 \quad b$ & 42,0 & d & 1,2 & $d$ \\
\hline PSR110 & 772 & c & $477 \quad b$ & $2,4 a$ & 40,0 & d & 1,2 & $d$ \\
\hline$\overline{C V}(\%)$ & 8,5 & & 12,4 & 13,5 & 3,3 & & 14,5 & \\
\hline
\end{tabular}

Médias seguidas de mesma letra nas colunas não diferem entre si a 5\% de probabilidade pelo teste de Scott-Knott;

${ }^{1}$ Escala visual de notas variando de 1 (folhas externas altamente infectadas por Bremia lactucae) a 5 (folhas externas sadias);

Os dados foram submetidos à análise de variância, seguindo esquema sugerido por Pimentel Gomes (1990), sendo as médias comparadas pelo teste de ScottKnott, ao nível de $5 \%$ de probabilidade.

\section{RESULTADOS E DISCUSSÃO}

A massa fresca total variou de 772 a 1268 g planta $^{-1}$ (Tabela 1). Os melhores desempenhos foram obtidos pelas linha- gens P1823-0014206-1 (1268 g planta $\left.{ }^{-1}\right)$ e P1823-0014325-3 (1232 g planta $^{-1}$ ), que não diferiram estatisticamente entre si. Numericamente esses resultados foram superiores aos obtidos por Salatiel et al. 
(2001), que com a cultivar Lorca, nas condições de Jaboticabal (SP), obtiveram 308,5 g planta $^{-1}$ e, semelhantes aos obtidos por Yuri (2000), para a cultivar Lucy Brown (972,5 g planta $\left.^{-1}\right)$, nas condições do município de Boa Esperança (MG).

A massa fresca comercial variou de 378 a 729 g planta $^{-1}$. Entre os melhores genótipos obteve-se variação de 561 a 729 g planta $^{-1}$, resultados semelhantes ao obtido por Yuri (2000), que nas condições de outono, em Boa Esperança, obteve massas frescas comerciais variando de 482 a 711 g planta $^{-1}$. Resultados similares verificados nesses trabalhos, provavelmente, são em função da época de plantio, tendo em vista o fato de a alface americana apresentar melhores produções nas condições de outono e inverno.

O comprimento do caule variou de 1,9 a $4,9 \mathrm{~cm}$. Entre os melhores genótipos para massa fresca comercial, vale destacar a cultivar Paloma, que com um comprimento de caule de $2,5 \mathrm{~cm}$, foi superior aos demais, não diferindo das linhagens RTP3030, PSR 110 , P1831-0015110-1, SVR1095, PSR 1115, P1871-2772 e P1871-2817. O comprimento do caule é uma característica importante, principalmente para alface destinada à indústria, devendo ser bastante reduzido, proporcionando menores perdas durante o processamento (Yuri et al., 2002). Na prática, caules com até $6,0 \mathrm{~cm}$ seriam os mais adequados, sendo ainda aceitáveis até o patamar de 9,0 cm e inaceitáveis ou menos recomendados para processamento acima deste índice. Dentro deste contexto, os demais genótipos com no máximo 4,9 cm, estão dentro de um limite aceitável, sendo inferiores ao obtido por Bueno (1998), que apresentou comprimentos de caule variando de 5,6 a $6,8 \mathrm{~cm}$.

A circunferência da cabeça comercial variou de 35,6 a 49,2 cm. Essa característica indica o tamanho do produto comercial, onde cabeças maiores proporcionam maiores rendimentos no beneficiamento. A linhagem P18230014325-3 com 49,2 cm, apresentou a maior circunferência e foi estatisticamente superior às demais. Esse resulta- do foi numericamente semelhante ao observado por Yuri (2000), que obteve para o melhor material, circunferência de $48,1 \mathrm{~cm}$.

$\mathrm{Na}$ avaliação da resistência ao fungo causador do "míldio", obteve-se notas de 1 a 5 (Tabela 1), onde os piores desempenhos foram apresentados pelas linhagens 99C962; SVR1095; PSR110; Ace; SVR1153; P1871-2788; P18712817; P1823-0014025-3; PSR1904; RTP2020 e RTP3030, com notas 1,0 ; 1,$0 ; 1,2 ; 1,2 ; 1,2 ; 1,2 ; 1,5 ; 1,5 ; 1,5 ; 1,5$ e 1,5 ; respectivamente. Por outro lado destacaram-se: SVR2210 (4,2); GX635 (4,2); P1843-1000148-1 (4,5); P18310015585-1 (4,5); P1831-0015338-1 (4,5); P1790-011945-1 (4,7); P18140013283-1 (4,7); P1843-1000697-1 (4,7); P1843-1000171-1 (4,7); P18431000179-2 (5,0); P1843-1000572-1 $(5,0)$; P1692-0001843-1 (5,0); P18310015110-1 (5,0); P1831-0015060-1 (5,0); P1831-0015583-2 (5,0); P18310015309-1 (5,0); P1831-0015058-2 $(5,0)$; P1823-0014206-1 (5,0); P18310015036-1 $(5,0)$ e P1823-0014325-3 $(5,0)$, sem diferirem estatisticamente entre si. A cultivar Raider, utilizada como padrão, mostrou-se altamente suscetível ao referido fungo.

Diante desses resultados e nas condições em que se realizou o experimento, vários genótipos tornam-se em opções de escolha pelos produtores durante o período de inverno, entre os quais: P1823-0014206-1; P1831-0015583-2; P1843-1000697-1; P1843-1000171-1; SVR2210; P1831-0015585-1; P18431000572-1; GX635; P1790-011945-1; P1692-0001843-1; P1831-0015058-2; P1831-0015338-1; P1831-0015309-1; P-1831-00150060-1; P1814-0013283-1 e P1823-0014325-3.

\section{LITERATURA CITADA}

BUENO, C.R. Adubação nitrogenada em cobertura via fertirrigação por gotejamento para a alface americana em ambiente protegido. (Tese mestrado), UFLA, Lavras, 1998, 54 p.

CONJUNTURAL de produtos por agência CEAGESP. Boletim Mensal. São Paulo. jan./dez. 2001
DAVIS, R.M.; SUBBARAO, K.V.; RAID, R.N.; KURTZ, E.A. Compendium of lettuce diseases. St. Paul: The American Phytopathological Society, 1997. 79 p.

DECOTEAU D.R.; RANWALA, D.; McMAHON M.J.; WILSON, S.B. The lettuce growing handbook: botany, field procedures, growing problems, and postharvest handling. Illinois: Oak Brook, 1995. 60 p.

FAO. Agricultural production, primary crops. Disponível em: <http://www.fao.org $>$. Acesso em 10/04/2002.

IBGE. Censo agropecuário: Sudeste. Rio de Janeiro, 1996. Disponível em <www.sidra.ibge.gov.br> Acesso em 13/03/00.

JACKSON, L.; MAYBERRY, K.; LAEMMLEN, F.; KOIKE, S.; SCHLUBACK, K. Iceberg lettuce production in California. Disponível em: <http// www.vegetablecrops.ucdavis $>$. Acesso em 24/10/99. KATAYAMA, M. Nutrição e adubação de alface, chicória e almeirão. In: SIMPÓSIO SOBRE NUTRIÇÃO E ADUBAÇÃO DE HORTALIÇAS, 1990, Jaboticabal. Anais...Piracicaba: POTAFOS, 1993. Cap.4, p.141-148.

MEIRELLES, J.C.S. Classificação de alface. São Paulo: Horti \& Fruti, 1998. (Folders).

MOTA, J.H. Efeito do cloreto de potássio via fertirrigação na produção de alface americana em cultivo protegido. Lavras. (Tese mestrado), UFLA, Lavras, 1999. 46 p.

MOTA, J.H.; YURI, J.E.; FREITAS, S.A.C.; RODRIGUES JUNIOR., J.C.; RESENDE, G.M.; SOUZA, R.J. Avaliação de cultivares de alface americana durante o verão em Santana da Vargem, MG. Horticultura Brasileira, Brasília, v.21, n.2, p.2344-237, 2003

MOTA, J.H.; YURI, J.E.; FREITAS, S.A.C.; RODRIGUES JUNIOR J.C.; RESENDE, G.M.; SOUZA, R.J. Comportamento de cultivares de alface americana quanto à queima dos bordos ("tip-burn") na região sul de Minas Gerais. Horticultura Brasileira, Brasília, v. 20, suplemento CD-ROM, julho 2002.

PERSLEY D. Diseases of vegetable crops. Queensland: Department of Primary Industries, 1994. 164 p.

PIMENTEL GOMES, F. Curso de estatística experimental. 13. ed. São Paulo: Nobel, 1990. 468 p. RIBEIRO, A.C.; GUIMARÃES, P.T.G.; ALVAREZ V.V.H. Recomendações para o uso de corretivos e fertilizantes em Minas Gerais. Viçosa: CFSEMG, 1999. 359 p. (5 a aproximação).

SALATIEL, L.T.; BRANCO, R.B.F.; MAY, A.; BARBOSA, J.C.; PAULA, C.M.; CECILIO FILHO, A.B. Avaliação de cultivares de alface em diferentes épocas de plantio, cultivadas em casa de vegetação. Horticultura Brasileira, Brasília, v. 19, suplemento CD-ROM, julho 2001.

YURI, J.E. Avaliação de cultivares de alface americana em duas épocas de plantio e dois locais do sul de Minas Gerais. Lavras. (Tese mestrado), UFLA, Lavras, 2000. 51 p.

YURI J.E.; MOTA, J.H.; SOUZA, R.J.; RESENDE, G.M.; FREITAS, S.A.C.; RODRIGUES JUNIOR, J.C. Alface americana: cultivo comercial. Lavras: UFLA, 2002. 51 p. Texto acadêmico. 